\title{
ON THE BLACKWELL PROPERTY OF LUZIN SETS
}

\author{
JAKUB JASIŃSKI
}

\begin{abstract}
We prove under the $\mathrm{CH}$ (Continuum Hypothesis) that there are strongly Blackwell Luzin sets and not Blackwell Luzin sets. We also indicate how these results can be generalized under MA (Martin's Axiom).
\end{abstract}

1. Let $X$ be a set and $C$ a $\sigma$-algebra of subsets of $X$. By $A t(C)$ we shall denote the family of all atoms of $C$. If $Y \subset X$, then by $C_{Y}$ we mean the $\sigma$-algebra $\{C \cap Y: C \in C\}$.

In this paper we shall deal only with the subsets of the real line $\mathbf{R}$, and by $B$ we denote the $\sigma$-algebra of all Borel subsets of $\mathbf{R}$.

Let us now recall the main definitions. An uncountable set $L \subset \mathbf{R}$ is called a Luzin set iff, whenever $F \subset \mathbf{R}$ is a meager set, $|F \cap L| \leq \omega$. It is well known that $\mathrm{CH}$ implies that there are Luzin sets (see J. C. Oxtoby [7, p. 78]).

The set $X \subset \mathbf{R}$ is called a Blackwell set iff whenever $C \subset B_{X}$ is a countably generated (c.g.) $\sigma$-algebra, which separates the points, then $C=B_{X}$.

The set $X \subset \mathbf{R}$ is called a strongly Blackwell set iff, whenever $C \subset B_{X}$ and $D \subset B_{X}$ are c.g. $\sigma$-algebras such that $A t(C)=A t(D)$, then $C=D$. It is well known that all analytic subsets of $\mathbf{R}$ are strongly Blackwell sets. For this, and for further discussion of these properties, see K. P. S. Bhaskara Rao and B. V. Rao [1, Chapter $2]$.

2. M. Orkin [6] proved that there is a Blackwell set $X$ such that neither $X$ nor $\mathbf{R} \backslash X$ contains an uncountable Borel set. Modifying a version of Orkin's method, presented in [1, Chapter 2, §9], we shall prove

THEOREM $1(\mathrm{CH})$. There exists a Luzin set $L$ such that if $L \subset L^{\prime}$ and $L^{\prime}$ is a Luzin set, then $L^{\prime}$ is a strongly Blackwell set.

Proof. Assume CH. Let

$$
F_{0} \subset F_{1} \subset \cdots \subset F_{\alpha} \subset \cdots, \quad \alpha<2^{\omega},
$$

be the sequence of meager $\mathcal{F}_{\sigma}$ sets such that for each meager set $X \subset \mathbf{R}$ there is an $\alpha<2^{\omega}$ such that $X \subset F_{\alpha}$, and let

$$
\left(C^{0}, B^{0}\right),\left(C^{1}, D^{1}\right), \ldots,\left(C^{\alpha}, D^{\alpha}\right), \ldots, \quad \alpha<2^{\omega},
$$

be the sequence of all ordered pairs $(C, D)$, where $C \subset D \subset B$ are c.g. $\sigma$-algebras, such that

$$
\text { for every } \alpha<2^{\omega}, \quad \operatorname{At}\left(C_{\mathbf{R} \backslash F_{\alpha}}\right) \neq A t\left(D_{\mathbf{R} \backslash F_{\alpha}}\right) \text {. }
$$

Since $C \subset D$ and both are c.g., (3) can be restated equivalently as follows: For every $\alpha<2^{\omega}$ there is an atom of $C_{\mathbf{R} \backslash F_{\alpha}}$, which contains two different atoms of $D_{\mathbf{R} \backslash F_{\alpha}}$.

Received by the editors January 3, 1984.

1980 Mathematics Subject Classification. Primary 28A05; Secondary 04A15.

(C) 1985 American Mathematical Society $0002-9939 / 85 \$ 1.00+\$ .25$ per page 
Now we shall construct the set $L=\left\{x_{\alpha}, y_{\alpha}: \alpha<2^{\omega}\right\}$ by the transfinite induction on $\alpha$. We pick $x_{0}, y_{0}$ belonging to one atom of $C^{0}$ and to two different atoms of $D^{0}$. Suppose we have chosen elements $x_{\beta}, y_{\beta}$ for $\beta<\alpha$. We pick $x_{\alpha}, y_{\alpha}$ belonging to one atom of $C_{\mathbf{R} \backslash F_{\gamma}}^{\alpha}$ and to two different atoms of $D_{\mathbf{R} \backslash F_{\gamma}}^{\alpha}$ where

$$
\gamma=\min \left\{\delta: \forall_{\beta}<\alpha, x_{\beta}, y_{\beta} \in F_{\delta}\right\} .
$$

$L$ is the Luzin set since for each meager set $X$ there is an $\alpha<2^{\omega}$ such that $L \cap X \subset L \cap F_{\alpha} \subset\left\{x_{\beta}, y_{\beta}: \beta<\alpha\right\}$.

Suppose that $L \subset L^{\prime}$ and $L^{\prime}$ is the Luzin set. We shall check that $L^{\prime}$ is a strongly Blackwell set. Notice (cf. [1, Proposition 8, p. 23]) that is suffices to show that whenever $C^{\prime} \subset D^{\prime} \subset B_{L^{\prime}}$ are c.g. $\sigma$-algebras and $\operatorname{At}\left(C^{\prime}\right)=\operatorname{At}\left(D^{\prime}\right)$ then $C^{\prime}=D^{\prime}$.

Let $C^{\prime} \subset D^{\prime} \subset B_{L^{\prime}}$ be c.g. $\sigma$-algebras with the same atoms and let $C \subset B$ and $D \subset B$ be c.g. $\sigma$-algebras on $\mathbf{R}$ such that $C \subset D$, and $C_{L^{\prime}}=C^{\prime}$, and $D_{L^{\prime}}=D^{\prime}$. Since $\operatorname{At}\left(C_{L^{\prime}}\right)=\operatorname{At}\left(D_{L^{\prime}}\right)$, there exists an $\alpha_{0}<2^{\omega}$ such that $\operatorname{At}\left(C_{\mathbf{R} \backslash F_{\alpha_{0}}}\right)=\operatorname{At}\left(D_{\mathbf{R} \backslash \boldsymbol{F}_{\alpha_{0}}}\right)$. Otherwise, the pair $(C, D)$ would appear in the sequence (2), and we could find in $L$ two different elements from one atom of $C$ which do not belong to one atom of $D$, but this contradicts $A t\left(C_{L^{\prime}}\right)=\operatorname{At}\left(D_{L^{\prime}}\right)$.

The set $\mathbf{R} \backslash F_{\alpha_{0}}$ is a Borel set, hence $C_{\mathbf{R} \backslash F_{\alpha_{0}}}=D_{\mathbf{R} \backslash F_{\alpha_{0}}}$. Consider the family $\mathcal{A}=\left\{A \in \mathcal{A}(\mathcal{C}): A \cap\left(L^{\prime} \cap F_{\alpha_{0}}\right) \neq \varnothing\right\} .\left|L^{\prime} \cap F_{\alpha_{0}}\right| \leq \omega$, so $|A| \leq \omega$, and hence

$$
C_{L^{\prime} \cap \cup \mathcal{A}}^{\prime}=D_{L^{\prime} \cap \cup \mathcal{A}}^{\prime}
$$

On the other hand, $L^{\prime} \backslash \bigcup A \subset \mathbf{R} \backslash F_{\alpha_{0}}$, so

$$
C_{L^{\prime} \backslash \cup \mathcal{A}}^{\prime}=D_{L^{\prime} \backslash \cup \mathcal{A}}^{\prime}
$$

Since $L^{\prime} \cap \bigcup A \in C^{\prime}$, by (4) and (5) we have $C^{\prime}=D^{\prime}$.

REMARK 1. Note that for each Borel set $B \in B$ of second category, $L \cap B \neq \varnothing$. Let $B \in B$ be of second category, and let $B_{1}, B_{2} \in B$ be disjoint sets of second category, and $B=B_{1} \cup B_{2}$. A pair $\left(\sigma(B), \sigma\left(B, B_{1}\right)\right)$ satisfies the condition (3), so there are points $x, y, \in L$ such that $x \in B_{1}$ and $y \in B_{2}$.

The sets $X, Y \subset \mathbf{R}$ are called non-Borel-separated iff there does not exist a Borel set $B \in B$ such that $B \cap X=\varnothing$ and $Y \subset B$. The disjoint non-Borel-separated sets were used by K. P. S. Bhaskara Rao and B. V. Rao [1, pp. 27, 28] to construct not Blackwell sets. We shall use this idea to prove

THEOREM 2. If there exists a Luzin set, then there also is a Luzin set which is not Blackwell.

Proof. Let $L$ be a Luzin set. Without loss of generality we may assume that $L \subset(0,1)$. There are pairwise disjoint uncountable sets $X_{\alpha}, \alpha<\omega_{1}$, such that $L=\bigcup_{\alpha<\omega_{1}} X_{\alpha}$. Since each subfamily of $B_{L}$ of pairwise disjoint uncountable sets is countable, there is $\alpha_{0}<\omega_{1}$ such that $X_{\alpha_{0}} \notin B_{L}$. It is clear, that $L \backslash X_{\alpha_{0}}$ and $X_{\alpha_{0}}$ are disjoint non-Borel-separated sets. $L^{\prime}=\left(L \backslash X_{\alpha_{0}}\right) \cup X_{\alpha_{0}}+1$ is a Luzin not Blackwell set (cf. $\left[1\right.$, p. $\left.28,6^{\circ}\right]$ ).

REMARK 2. Slightly modifying this proof, one can show (under $\mathrm{CH}$ ) that every Borel set of second category contains a Luzin set which is not Blackwell.

The uncountable set $S \subset \mathbf{R}$ is called a Sierpinski set iff whenever $N \subset \mathbf{R}$ is of Lebesgue measure 0 , then $|S \cap N| \leq \omega$ (see J. C. Oxtoby [7, p. 78]). It is easy to 
see that considering in place of $(1)$ a sequence of $\mathcal{G}_{\delta}$ sets of Lebesgue measure 0 we obtain analogous (to Theorems 1 and 2) results about the Sierpiński sets.

3. The set $X \subset \mathbf{R}$ of cardinality $2^{\omega}$ is called the generalized Luzin (resp. Sierpiński) set iff whenever $Y \subset \mathbf{R}$ is a meager (resp. of Lebesgue measure 0) set then $|Y \cap X|<2^{\omega}$. It is well known that MA implies the existence of such sets (see D. A. Martin and R. M. Solovay [5]).

LEMMA 1 (MA). Let $X \subset \mathbf{R}$, and let $C$ be a c.g. $\sigma$-algebra of subsets of $X$. If $|\operatorname{At}(\mathrm{C})|<2^{\omega}$, then for each $\mathcal{E} \subset \operatorname{At}(\mathrm{C}), \cup \mathcal{E} \in C$.

PROOF. If $Y \subset \mathbf{R},|Y|<2^{\omega}$, then it follows from MA that $B_{Y}=P(Y)$ (see $[5])$, so to complete the proof it suffices to apply the characteristic function of a sequence of sets (see E. Marczewski [4]).

LEMMA 2 (MA). Let $S$ be a generalized Sierpinski set, and let $C \subset B_{S}$ be a c.g. $\sigma$-algebra. If $Y \subset S$ and $|Y|<2^{\omega}$, then there is a set $C \in C$ such that $Y \subset C$ and $C$ is a union of less than $2^{\omega}$ atoms of $C$.

This Lemma is due to E. Grzegorek.

PROOF. Without loss of generality we may assume that $S \subset(0,1)$ and $C$ separates the points of $S$. Let $\mu$ be a Lebesgue measure restricted to the $\sigma$-algebra C. For $Y^{\prime} \subset Y$ we define $\mu_{Y}\left(Y^{\prime}\right)=\inf \left\{\mu(C): C \in C \& Y^{\prime} \subset C\right\} . \mu_{Y}$ is a measure on $C_{Y}$ (see E. Marczewski [3]). Lemma 1 implies that $C_{Y}=P(Y)$ so, by MA, $\mu_{Y}(Y)=0$. Hence, there is $C \in C$ such that $\mu(C)=0$, so $|C|<2^{\omega}$.

LEMMA 3 (MA). Let $X \subset \mathbf{R}$ be such that each subfamily of $B_{X}$ of pairwise disjoint sets of cardinality $2^{\omega}$ is countable, and let $C \subset B_{X}$ be a c.g. $\sigma$-algebra separating the points of $X$. If $Y \subset X,|Y|<2^{\omega}$ then

$$
J=\left\{Y^{\prime} \subset Y: \exists C \in C\left(Y^{\prime} \subset C \&|C|<2^{\omega}\right)\right\}
$$

is an $\omega_{1}$-saturated $\sigma$-ideal such that $P(Y) / J$ is atomless.

PROOF. It is clear that $J$ is $\sigma$-additive and since $|Y|$ does not carry a nontrivial two-valued measure, $P(Y) / J$ is atomless. Suppose $Y_{\alpha} \subset Y, \alpha<\omega_{1}$, are pairwise disjoint . By MA, $C_{Y}=P(Y)$, so there are pairwise disjoint $B_{\alpha} \in C$ such that $B_{\alpha} \cap Y=Y_{\alpha}$. Hence, there is an $\alpha_{0}<\omega_{1}$ such that $\left|B_{\alpha_{0}}\right|<2^{\omega}$ and $Y_{\alpha_{0}} \in J$.

COROLlaRY 1 (MA). Assume that there are no nontrivial $\omega_{1}$-saturated $\sigma$ ideals such that the quotient algebra is atomless on sets of cardinality less than $2^{\omega}$. Let $L \subset \mathbf{R}$ be a generalized Luzin set and let $C \subset B_{L}$ be c.g. $\sigma$-algebra. If $Y \subset L$ and $|Y|<2^{\omega}$, then there is $C \in C$ such that $Y \subset C$ and $C$ is a union of less than $2^{\omega}$ atoms of $C$.

The proof is a straightforward application of the characteristic function of the sequence of sets (see [4]) and Lemma 3.

Using Lemmas 1 and 2 it is easy to check that, under MA, the method of proof of Theorem 1 provides an example of the generalized Sierpiński set, which is strongly Blackwell. To get this result for generalized Luzin sets we need an additional assumption as in Corollary 1 (see R. M. Solovay [8]). Let us only recall that this is true if there are no weakly inaccessible cardinals less than $2^{\omega}$.

Note that the proof of Theorem 2 stated for generalized Luzin and Sierpinski sets is just the same as the presented one. 
REMARK 3. It is clear that, under MA, sets of cardinality less than $2^{\omega}$ are strongly Blackwell (Lemma 1) and universally null. Since Luzin sets have strong measure 0 (so called property (C), see K. Kuratowski [2, Chapter III, §40, VII]) Theorem 1 provides, under $\mathrm{CH}$, an answer to the question of R. M. Shortt, "Can one construct, in ZFC, an uncountable universally null Blackwell space?" which could be found on the list of questions during the conference on Measure Theory in Oberwolfach in 1983. sion.

The author wishes to express thanks to Edward Grzegorek for a fruitful discus-

ADDED IN PROOF. Recently J. Pawlikowski has pointed out that the additional assumption in Corollary 1 is not essential hence that the following theorem is true: Assume MA. Then there exists a generalized Luzin set $L$ such that if $L \subseteq L^{\prime}$ and $L^{\prime}$ is a generalized Luzin set, then $L^{\prime}$ is strongly Blackwell.

\section{REFERENCES}

1. K. P. S. Bhaskara Rao and B. V. Rao, Borel spaces, Dissertationes Math. (Rozprawy Mat.) 190 (1981), 1-63.

2. K. Kuratowksi, Topology I, Academic Press, New York; PWN, Warsaw, 1966.

3. E. Marczewski, $O$ zbiorach $i$ funkcjach bezwzglednie mierzalnych, Odbitka ze sprawozdań z posiedzeń Towarzystwa Naukowego Warszawskiego 30 (1937), Wydzial III, 1-30 (English transl. by J. C. Morgan is available in manuscript).

4. 31 (1938), 207-223.

5. D. A. Martin and R. M. Solovay, Internal Cohen extensions, Ann. Math. Logic 2 (1970), 143178.

6. M. Orkin, A Blackwell space which is not analytic, Bull. Acad. Polon. Sci. 20 (1972), 437-438.

7. J. C. Oxtoby, Measure and category, Springer-Verlag, New York, 1971.

8. R. M. Solovay, Real-valued measurable cardinals, Axiomatic Set Theory, Proc. Sympos. Pure Math., vol. 13, Part 1, Amer. Math. Soc., Providence, R.I., 1971, pp. 397-428.

Institute of Mathematics, University of Gdańsk, Ul. Wita Stwosza 57, 80-952 GDAŃSK, POLAND 